Fuensanta Garrido Domené, Los teóricos menores de la Música Griega. Euclides el Geómetra, Nicómaco de Gerasa y Gaudencio el Filósofo, Barcelona: Cerix (2016), 516 páginas.

\author{
Laura Carolina Durán*
}

Para los estudiosos e interesados en la investigación de las fuentes musicales de la Antigüedad, esta reciente publicación no puede menos que ser gratamente recibida, dado que viene a suplir la carencia de traducciones castellanas que hay todavía hoy de importantes textos sobre la materia.

La autora, Fuensanta Garrido Domené, es Doctora en Filología por la Universidad de Murcia y ha publicado numerosos artículos y trabajos en colaboración sobre música antigua, una de sus principales áreas de estudio. Este libro, producto de su tesis de doctorado, ampliada para esta publicación, ha sido editado por la joven Editorial Cerix para su colección Harmonices Mundi.

La obra presenta la traducción al castellano de los textos griegos Sectio Canonis de Euclides, Manual de harmónica y Excerpta ex Nicómaco de Nicómaco de Gerasa y la Introducción a la harmónica de Gaudencio, acompañada de información preliminar, notas y comentarios. El texto está organizado en una introducción general; un índice de las abreviaturas, referencias de autores y ediciones utilizadas para la traducción; tres capítulos dedicados a Euclides, Nicómaco y Gaudencio; y finalmente un breve epílogo. Se incluyen un índice onomástico y un exhaustivo índice de términos musicales, matemáticos y astronómicos, que incorpora los vocablos en griego, acompañados de la versión latina de Meibom (primer editor de estas obras en latín, en 1652; cabe aclarar que esta edición se utiliza en este apartado, dado que para las traducciones de los textos completos la autora sigue las ediciones de K. von Jan, de 1895, re-edición 1995) y la versión castellana elegida, con la indicación del apartado del tratado respectivo donde los términos aparecen, aunque no la página. El volumen culmina con una lista de la bibliografía empleada, organizada en referencias generales, ediciones utilizadas para la traducción, y estudios específicos sobre cada uno de los autores abordados.

\footnotetext{
* Universidad Nacional de San Martín, Argentina.
} 
La introducción expone sumariamente las dos posiciones musicales antiguas conocidas: la pitagórica y la aristoxénica. La autora realiza una consideración de la naturaleza de estos tratados breves, que presentan los tópicos musicales básicos en forma de breviario, libres de cualquier aspecto problemático o dificultad crítica -cuestiones sí abordadas en la tratadística mayor de la que estos derivan-. Se agregan algunas anotaciones sobre cada una de las obras traducidas: la información sobre la datación, ediciones utilizadas, una caracterización general y presentación del programa de cada tratado, todos elementos que se desarrollarán con amplitud en los capítulos dedicados a los autores trabajados. Se anticipa la importancia de la figura de Nicómaco, así como el lugar clave que ocupan sus textos como eslabón entre el período clásico de la teoría musical y las posteriores obras medievales.

Aunque la presente edición no es bilingüe, el texto está poblado de referencias a locuciones griegas y latinas, y aclaraciones que fundamentan la elección terminológica realizada en castellano. También se hace referencia a las discusiones de traducción de los términos en otras lenguas modernas, de manera que quien lee, aún sin disponer del texto en su lengua original, tiene una abundante información al respecto, que se suma al trabajo presentado en el completo índice de términos musicales, matemáticos y astronómicos que mencionáramos supra.

Los capítulos de cada uno de los autores seleccionados tienen una estructura similar: una introducción, cuestiones relativas a autoría y datación, programa y fuentes del texto, influencia posterior, la traducción, y un comentario exhaustivo de cada obra.

El primer capítulo está dedicado a Euclides el Geómetra y su obra Sectio Canonis, pequeño tratado que versa sobre la relación entre principios matemáticos y verdades acústicas, inscripto en la tradición pitagórica. La autora introduce el texto de Euclides con la información de las tres tradiciones de transmisión del escrito (Cleónides, Porfirio y Boecio), y un copioso informe de las discusiones actualizadas en cuanto a datación y autoría, dado que no faltan críticos que cuestionan la atribución del texto al geómetra. Con respecto a la estructura de la obra, la autora distingue un proemio inicial, en el que primero se definen las bases físicas del sonido -aspecto con ecos del tratado aristoxénico y las consideraciones aristotélicas-, seguido por la introducción de los fundamentos matemáticos de los intervalos musicales, ahora sí, con claras referencias pitagóricas. Siguen al proemio las veinte proposiciones matemático-musicales, a su vez divididas en problemas y teoremas. Los teoremas I-IX son puramente matemáticos, y, a 
partir del X, se presentan los corolarios musicales. En esta introducción previa a la traducción del tratado, Garrido Domené incluye abundantes notas a pie con explicaciones a cada texto mencionado como fuente o influencia, de modo de orientar al lector que las desconozca.

La traducción propiamente dicha está precedida de un esquema que presenta, a modo de índice, la disposición de contenidos de la obra. De las 59 páginas destinadas a este primer tratado, 10 corresponden a la traducción; el resto se distribuyen entre la introducción mencionada y el extenso y clarificador comentario posterior. Éste cuenta con una introducción, centrada en el proemio, que ahonda en las raíces peripatéticas y platónicas, en la que, cabe resaltar, se hacen explícitos ciertos problemas de la concepción matemática de la música, al tiempo que se apela al sentido de la audición para la intelección de algunas cuestiones. Puede considerarse este señalamiento como una clave de lectura que advierte al lector sobre esta tensión entre un abordaje de la disciplina musical como puramente intelectiva, que sin embargo termina apelando a la sensibilidad auditiva. Subrayar esta discordancia que podría pasar desapercibida es un mérito no menor de la autora. Es esta una cuestión central en la clasificación de la música en tratados posteriores que se nutren directamente de estos textos. Recordemos sólo a modo de ejemplo la tripartición de la música en Boecio en musica mundana o música de las esferas, musica humana o música del alma y del cuerpo y musica instrumentalis o música vocal e instrumental. Por último, la autora analiza los teoremas que versan sobre los intervalos matemáticos. Se presenta una agrupación de estos teoremas, atendiendo a los tipos, definiciones y relaciones; y luego se estudia cada uno de manera particular. Garrido Domené acompaña sus análisis con ejemplos de cálculos, en los que se evidencia la necesidad del geómetra de encontrar como resultados números enteros, dado el desconocimiento que el mundo griego tenía de los decimales. Estas ejemplificaciones ayudan a comprender con mayor claridad los teoremas del tratado, que algún lector podría considerar tediosos y hasta confusos en relación con el objetivo del argumento, tal como sucede con la explicación de las notas musicales en sus nombres originarios y en la composición de las proporciones. En la explicación de los teoremas musicales, se incluyen observaciones relativas a la teoría musical de la época (sistema tonal de dos octavas, consonancias atribuidas a ciertos intervalos) que sirven de marco de referencia general de las cuestiones abordadas y esclarecen el entendimiento del texto euclidiano.

El capítulo central está dedicado a Nicómaco de Gerasa, de quien se presentan dos textos: Manual de harmónica y Excerpta ex Nicómano. A lo largo del 
texto se encuentra el término "harmonía" y sus derivados manteniendo la $h$, así se seńala la forma etimológica griega, dado que para los autores helenos esta voz se refería al ajuste o afinación de los distintos grados de la escala; concepto muy diferente de la moderna noción de armonía, que se refiere a la emisión simultánea de varios sonidos musicales. Tal como fuimos advertidos en la introducción general del libro, Nicómaco es quien recibe mayor atención (páginas 85 a 459). El estudio introductorio presenta datos sobre la biografía de Nicómaco; la época, y tradición de pensamiento; todas sus obras, tanto las conservadas como aquellas de las que sólo poseemos alguna mención en autores posteriores; y su reputación, influencia y transmisión posterior. Una vez más, cada autor de la tradición citado está referenciado en notas a pie, ubicadas en la misma página del cuerpo del texto. Conviene resaltar que la extensión dedicada a Nicómaco, que podría parecer desmedida, tiene plena justificación. Se trata de un autor de máxima influencia en la temática, y en toda la tradición neoplatónica, tal como podemos apreciarlo en la afirmación de Proclo de ser su reencarnación, o de Isidoro de Sevilla, quien lo equipara al mismo Pitágoras.

A partir del Manual de harmónica queda clara la filiación de Nicómaco al pensamiento pitagórico. En efecto, se ha considerado que la desconocida destinataria del manual era una pitagórica, y que el texto mismo era un panfleto pitagórico, más que propiamente un tratado. El Manual está compuesto por epístolas, que el autor escribe mientras realiza un incómodo viaje, organizadas en doce capítulos, cada uno encabezado por un descriptivo epígrafe que adelanta la materia a tratar.

El pormenorizado comentario de esta obra es el apartado más extenso del libro. Contamos con un pequeño resumen de la temática de cada capítulo y luego el análisis de su contenido, con numerosas aclaraciones terminológicas y los giros intertextuales presentes. Señala acertadamente la autora del comentario que, si bien el clima es claramente pitagórico, hay elementos aristoxénicos en la obra, que Nicómaco no se ocupa de mencionar-como los tipos de voz, la definición de nota y otros-. Resaltemos ciertos capítulos que evidencian la importancia de esta obra: el tercero presenta la teoría de la música de las esferas y puede considerarse la versión más antigua de este concepto -luego de las referencias platónicas en República X y Timeo-, basándose Nicómaco en fuentes de las más antiguas (para nosotros desconocidas dado que no se conservaron), de acuerdo al criterio unificado de la crítica; el sexto presenta la primera versión de la "leyenda de los martillos pitagóricos", examinada en el comentario en sus más ínfimos detalles; y el capítulo nueve presenta la transmisión de un fragmento de 
Filolao, quien según Nicómaco fue seguidor directo de Pitágoras y el primero en transmitir por escrito algo de lo dicho por aquel. Las comparaciones de las consideraciones musicales con nuestra actual escala y sistema de notación musical facilitan cabalmente la comprensión del texto geraseano.

El segundo texto del de Gerasa que se traduce en este volumen es el conocido como Excerpta ex Nicómaco, una recopilación de diez fragmentos que en líneas generales retoman lo expuesto en el Manual. En su análisis, la autora procede de la manera que ya hemos descripto, remarcando particularmente la influencia del tratado en la tradición posterior, especialmente en Boecio.

El tercer y último capítulo está dedicado a Gaudencio el Filósofo, de quien se presenta su Introducción a la harmónica. El esquema de abordaje es el mencionado. La obra de Gaudencio cuenta con un proemio y veintitrés capítulos, que la traductora se esfuerza en agrupar, dada la diversidad de los mismos, del siguiente modo: I-IX aristoxénicos, X-XVI pitagóricos, XVII-XIX nuevamente aristoxénicos, y finalmente XX-XXIII notación musical griega. El proemio del texto gaudenciano está precedido por el famoso epígrafe "canto para entendidos; cerrad las puertas, profanos", elemento que permite a la autora ubicar componentes de la tradición pitagórica y órfica, para luego dar comienzo a la exégesis del apartado programático, donde se mencionan las categorías que se estudiarán y cómo se enlazan una con otra: "notas, intervalos, escalas, tonos, modulaciones y melopeyas en todos los géneros de la Harmónica”. Es de destacar que estas siete categorías tradicionales de la ciencia harmónica son de inspiración aristoxénica y se encuentran en numerosos autores. Resaltemos que en el capítulo dos las propiedades atribuidas a las notas - matiz, posición y duración- no han sido enumeradas con anterioridad a Gaudencio, lo cual representa un grado de originalidad de este autor. El comentario trae nuevas referencias a lo que ya mencionáramos sobre las tensiones entre la comprensión matemática y la apelación al oído como el órgano que reconoce y diferencia los intervalos melódicos y consonantes de los que no lo son. Nuevamente, en la explicación del sistema de escalas antiguo, se ofrece un modelo en nuestra notación actual, lo que simplifica la lectura. El esclarecimiento de los problemas que genera la división del tono, y por tanto el uso de dos tipos de semitonos leimma y apotomé, permite entender con precisión este dificultoso tema, al tiempo que da mayor valor al texto de Gaudencio, que es uno de los pocos que transmite los signos musicales antiguos. 
Para un lector que se acerca por primera vez a un tratado musical antiguo el texto puede resultar algo complejo, dada la dificultad misma de la materia y las numerosas referencias terminológicas y a múltiples autores; sin embargo, esto último hace, precisamente, a la riqueza del material que se nos presenta. Otros inconvenientes que puede encontrar algún lector son algunas expresiones de difícil interpretación y algunas erratas: extensos pasajes en latín sin traducir (pp. 248, 266 y 415), la fórmula de frecuencia del sonido en el comentario sobre el Manual de Nicómaco (p. 204), la repetición de la explicación simbólica del número siete (p. 206), “Jubal” por "Tubal” en el mismo capítulo (p. 220), la discusión sobre si debe considerarse a Filolao seguidor o sucesor de Pitágoras (p. 252 y nota 590), algunos errores de tipeo (p. 397 "híapte” por "hípate”, p. 448 "greiegos" por "griegos"), libros citados en el trabajo que no son consignados en la lista de bibliografía. Algunos especialistas podrían llegar a sentir alguna incomodidad ante la permanente referencia al pitagorismo como "secta", nominación con la que no acordaría gran parte de la crítica.

Con todo, ante la nimiedad de estos detalles, no puede más que agradecerse esta primera versión castellana de estos estimulantes textos y el arduo trabajo de contextualización y exégesis textual que ha realizado Garrido Domené.

\section{(c) $(\mathrm{D}) \Theta$}

ENDOXA está bajo una licencia de Creative Commons Reconocimiento-NoComercial-SinObraDerivada 4.0 Internacional 